\title{
L1 Adaptive Control for Wind Gust Rejection in Quad-Rotor UAV Wind Turbine Inspection
}

\author{
Ramón A. Suárez Fernández, Sergio Dominguez, and Pascual Campoy
}

\begin{abstract}
This work presents preliminary results of the control method developed for autonomous inspection of wind turbines. High wind gusts are a major deterrent of outdoor Unmanned Aerial Vehicle (UAV) operations, in which, common classical control methods such as Proportional-IntegralDerivative (PID) control do not perform well. Therefore, more robust adaptive control methods must be employed. We propose the use of an $\mathcal{L}_{1}$ adaptive velocity controller that is capable of fast adaptation and guaranteed robustness while withstanding any disturbances encountered during flights. Considerable increase in performance of the $\mathcal{L}_{1}$ adaptive controller is demonstrated by comparing the proposed approach to a Linear Quadratic Regulator (LQR) method in simulation and a benchmark PID controller in several real flight experiments with added wind gusts.
\end{abstract}

\section{INTRODUCTION}

\section{A. Motivation}

The construction of wind farms on a global scale has taken place in the last 20 to 25 years. The lifespan of these farms has extended beyond initial estimates, hence, there is increasing work focused on prolonging the durability of active wind turbines. This is achieved, in part, by optimizing and extending inspection and maintenance tasks. These tasks have had an economic impact in this sector due to the fact that energy production must be interrupted for technicians to have a safe working environment. For the most part, these machines operate in remote facilities with difficult access, where damages have commonly been assessed by means of simple ground based vision systems or more dangerous rope and platform access.

Therefore, new inspection technologies for wind turbines that have the capability of detecting damages on an early stage are being extensively researched. This would allow operators to integrate any repairs needed into the normal maintenance plans of the wind farms without having an impact on production. Among inspection technologies that are intended, the most sought after are those which do not require the wind turbines to stop and effectively eliminate production losses.

The use of Quad-Rotor Unmanned Aerial Vehicles (UAVs) for inspection has been a widely emerging market in recent years with many applications, such as power line inspection

This work was performed during the AEROS project, financed by the program 2014 Retos-Colaboración del Programa Estatal de Investigación, Desarrollo e Innovación Orientada a los Retos de la Sociedad, supported by the Ministerio de Economía y Competitividad, of Spain ref: RTC-20141977-3. As well as MICYT DPI2014-60139-R.

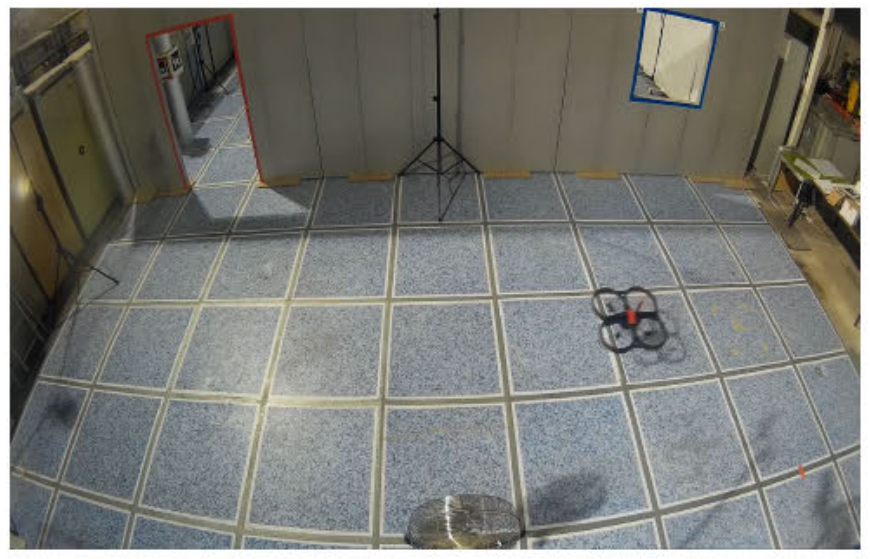

Fig. 1: Quad-Rotor flying into the fans airflow during one of the step reference real flight tests.

(i.e. see [8] and [17]). These vehicles allow fast and lowpriced on-site analysis while lowering the risks on human operators. Quad-Rotor UAV units can also be equipped with a wide variety of sensing equipment, such as High-Definition cameras, Thermal-Imaging cameras, and Light Detection and Ranging (LIDAR) sensors which can optimize the detection of failures in the blades caused by erosion, fissures, lightning damage on arrestors, etc.

Even though Quad-Rotor UAVs have been shown to be suitable for wind turbine inspection, when flown in semiautonomous or manual mode, limitations still arise when attempting fully autonomous missions. Problems in these high risk scenarios, such as significant wind gusts due to atmospheric turbulence or actuator failure are a major deterrent of outdoor aerial vehicle operations. Therefore, minimizing the effects of wind gust disturbances on the vehicles and stabilizing properly in these scenarios would increase the scope of potential applications for Quad-Rotor UAVs [2].

In order to minimize these effects, precise control is of foremost importance. Nevertheless, commonly widespread solutions for control (e.g. Proportional-Integral-Derivative (PID)) generally do not meet the performance requirements when subject to wind gust disturbances [18]. Hence, to execute the desired control over the Quad-Rotor UAV efficiently while withstanding environmental disturbances, robust adaptive control methods are required.

Adaptive control strategies allow for applications in which environmental or dynamic conditions can change mid-flight by automatically adjusting control structures or parameters 
[15]. These changes can be abrupt and thus, the adaptation method must be fast yet robust. Model Reference Adaptive Control (MRAC) methods can be used for such applications but can be particularly susceptible to time delays [1]; Thus, an advanced version of MRAC, termed $\mathcal{L}_{1}$ Adaptive Control was developed to address these issues [5].

$\mathcal{L}_{1}$ adaptive control, first proposed by $\mathrm{Cao}$ and Hovakimyan [4], is well known for its guaranteed robustness while achieving fast adaptation [7], [20]; This differs from the past generation of adaptive control laws with oscillatory adaptation behaviors, which can cause oscillation of the UAV [13]. It's main strength is the capability of fast adaptation with guaranteed low-frequency control signal. This provides the desired transient performance for both the systems signals, input and output, simultaneously, while the low-pass filter in the feedback loop attenuates the high frequency components in the control signal [5].

\section{B. Related Works}

Control of UAVs in the presence of high wind gusts, or during fast and aggressive maneuvers has been brought up by a number of recent papers, [25], [31]. In [27], the results of an Incremental Nonlinear Dynamic Inversion (INDI) gust resistant position controller tested with wind gusts produced by an industrial fan, are shown. Currently, many methods developed by researchers opt for using on-board wind sensors to estimate the wind forces [28]. Nonetheless, these methods add unwanted costs and complexities to the system. Another common approach is to estimate the wind velocity based on a system model [31], [24], [26]. The downside of this method is that it's very dependent on a precise model with no adaptation for unknown parameters.

Other control techniques for precise and robust control employing non-linear control theory include; Feedback Linearization [9], Sliding Mode [14] and Backstepping Control [16]. However, the effectiveness managed by employing non-linear methods of control depend on the accuracy of the dynamic model of the system. To improve dynamic parameters, non-linear control methods need more precise models and a auxiliary augmentation system for handling unpredictable uncertainties [13].

$\mathcal{L}_{1}$ Adaptive control for Quad-Rotor UAVs has been applied by various researchers with promising results [29], [15]. In [16], the authors use the backstepping technique and augment it with the $\mathcal{L}_{1}$ adaptive controller while the authors in [19] combined the theory with neural network control to produce attitude controllers. A common feature can be seen in these studies, that is, the disturbance on the UAVs output gain is either combined with other adaptive parameters or assumed to be known.

\section{Contributions and Outline}

In this work, an $\mathcal{L}_{1}$ adaptive controller is implemented for $x$ and $y$ (forward and lateral) velocity that maintains the desired reference even in the presence of wind gust disturbances. Experiments have been performed to test the proposed solutions in simulation and validation was made with real flights using a fans airflow as disturbance. Results are then compared to a Linear Quadratic Regulator (LQR) controller in simulation and a benchmark PID controller in real flights.

The remainder of the paper is organized as follows: Section II presents the Quad-Rotor UAV dynamic model; Section III shows the $\mathcal{L}_{1}$ adaptive output feedback control theory and equations. Section IV focuses on the simulation experiments and results while Section V presents the real flight experiments and results; Lastly, Section VI concludes the paper and details future works.

\section{QUAD-Rotor UAV Dynamic Model}

In this section, the dynamics of the Quad-Rotor UAV are explained. Specifically, the reference model used in the $\mathcal{L}_{1}$ adaptive control method in Section III is based on the linearized Quad-Rotor dynamics and the state-space model. The dynamic model considered is of a four fixed-pitch rotor UAV as seen in Fig. 2. The modeling of such UAVs has been extensively studied in detail by numerous research groups such as the works of [3] and [10].

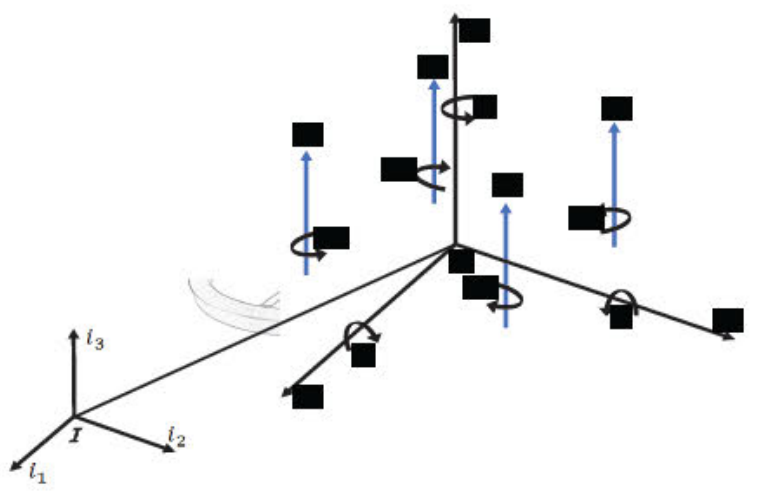

Fig. 2: Quad-Rotor configuration used for dynamic model. The body-fixed reference frame $\mathcal{B}$ is attached to the center of mass of the Quad-Rotor with $b_{1}$ pointing towards the East axis ( $x$ direction), $b_{2}$ pointing towards the North axis ( $y$ direction) and $b_{3}$ pointing upwards ( $z$ direction).

\section{A. Rigid-Body Model}

Let $\tau_{\phi}, \tau_{\theta}$ and $\tau_{\psi}$ be the roll, pitch and yaw moments; and $F_{z}{ }^{b}$ the thrust force generated by the four propellers, then, the simplified model (neglecting forces due to moments of inertia, angular velocities of the blades and assuming low speeds) of the Quad-Rotor UAV is expressed as:

$$
\left\{\begin{aligned}
I_{x} \dot{\omega}_{x} & =\left(I_{y}-I_{z}\right) \omega_{y} \omega_{z}+\tau_{\phi} \\
I_{y} \dot{\omega}_{y} & =\left(I_{z}-I_{x}\right) \omega_{x} \omega_{z}+\tau_{\theta} \\
I_{z} \dot{\omega}_{z} & =\left(I_{x}-I_{y}\right) \omega_{x} \omega_{y}+\tau_{\psi} \\
m \ddot{x} & =F_{z}{ }^{b}(\cos \phi \sin \theta \cos \psi+\sin \phi \sin \psi) \\
m \ddot{y} & =F_{z}{ }^{b}(\cos \phi \sin \theta \sin \psi-\sin \phi \cos \psi) \\
m \ddot{z} & =F_{z}{ }^{b}(\cos \phi \cos \theta)-m g
\end{aligned}\right.
$$


with

$$
\left\{\begin{array}{c}
F_{z}{ }^{b}=\left(F_{1}+F_{2}+F_{3}+F_{4}\right) \\
\tau_{\phi}=\left(F_{2}+F_{3}-F_{1}-F_{4}\right) \cdot l \\
\tau_{\theta}=\left(F_{3}+F_{4}-F_{1}-F_{2}\right) \cdot l \\
\tau_{\psi}=M_{1}-M_{2}+M_{3}-M_{4}
\end{array}\right.
$$

where $F_{1-4}$ and $M_{1-4}$ are the forces and moments generated by each rotor respectively. In (1)-(6) $x, y$ and $z$ are the position of the center of mass of the quad-rotor in the inertial frame of reference; $\phi, \theta$ and $\psi$ are the Euler angles in the inertial frame of reference; $\omega_{x}, \omega_{y}$ and $\omega_{z}$ are the angular velocities in the body-fixed frame of the propeller blades; $I_{x}, I_{y}$ and $I_{z}$ are the moments of inertia; and $m$ and $l$ are the mass and distance from the rotors to the center of mass respectively.

\section{B. Linearized Model}

The linear model of the quad-rotor is calculated around a hovering point, this implies an arbitrary position $x=x_{0}$, $y=y_{0}, z=z_{0}$ and heading $\psi=\psi_{0}$, with $\theta=\phi=\dot{\theta}=$ $\dot{\phi}=\dot{\psi}=\dot{x}=\dot{y}=\dot{z}=0$. The transformation of the angular velocities from the inertial frame to the body frame can be obtained by [2]:

$$
\left[\begin{array}{c}
\omega_{x} \\
\omega_{y} \\
\omega_{z}
\end{array}\right]=\left[\begin{array}{ccc}
1 & 0 & -\sin \theta \\
0 & \cos \phi & \sin \phi \cos \theta \\
0 & -\sin \phi & \cos \phi \cos \theta
\end{array}\right]\left[\begin{array}{c}
\dot{\phi} \\
\dot{\theta} \\
\dot{\psi}
\end{array}\right]
$$

Since at hover $\theta=\phi=0$, the trigonometric functions sine and cosine can be approximated as $\cos (k) \approx 1$ and $\sin (k) \approx k$ for $k=\{\phi, \theta\}$. Therefore (8) can be expressed as:

$$
\left\{\begin{array}{c}
\omega_{x}=\dot{\phi}-\theta \dot{\psi} \\
\omega_{y}=\dot{\theta}+\dot{\psi} \phi \\
\omega_{z}=-\dot{\theta}+\dot{\psi}
\end{array}\right.
$$

Assumption 1: Near hover, the product of an angle and an angles derivative is very small and can be approximated to zero.

Therefore, around hover, the angular velocity components are approximately the time derivative of the Euler angles:

$$
\left\{\begin{array}{l}
\omega_{x}=\dot{\phi} \\
\omega_{y}=\dot{\theta} \\
\omega_{z}=\dot{\psi}
\end{array}\right.
$$

Using (10) and Assumption 1, the system in (1)-(3) can be rewritten as:

$$
\ddot{\phi}=\frac{\tau_{\phi} \cdot l}{I_{x}}, \quad \ddot{\theta}=\frac{\tau_{\theta} \cdot l}{I_{y}}, \quad \ddot{\psi}=\frac{\tau_{\psi}}{I_{z}} .
$$

Assumption 2: Attitude dynamics are controlled independently, therefore, the yaw rate and the $x$-y position can be decoupled from each other. This is valid for small rates of yaw.
Taking into account Assumption 2 , the value for trigonometric functions at hover point and letting $F_{z}{ }^{b}=m g+\Delta F_{z}{ }^{b}$, the system in (4)-(6) linear dynamics can be written as:

$$
\ddot{x}=g \theta, \quad \ddot{y}=-g \phi, \quad \ddot{z}=\frac{\Delta F_{z}^{b}}{m}
$$

\section{State-Space Model Representation}

In order to implement the controller, the previous equations of the system must be rewritten as a state-space model. Since (11) and (12) are approximately decoupled about each attitude axis, the control inputs $\tau_{\phi}, \tau_{\theta}$ and $\tau_{\psi}$ can be implemented independently. This is an accurate assumption when the angular velocities and the attitude angles are small [11].

Therefore, the state-space model for the system will be separate for each control input i.e. $\left(\tau_{\phi}, \tau_{\theta}, \tau_{\psi}\right.$ and $\left.F_{z}{ }^{b}\right)$. In (13) - (16) the decoupled state-space model of the pitch and roll subsystems are presented which will be used for the control design methods in the following Sections.

1) Pitch Subsystem:

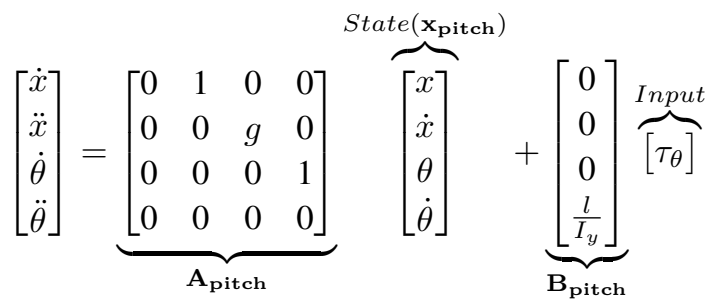

$$
\begin{aligned}
& y=\underbrace{\left[\begin{array}{llll}
0 & 1 & 0 & 0
\end{array}\right]}_{\mathbf{c}_{\text {pitch }}} \mathbf{x}_{\text {pitch }}
\end{aligned}
$$

2) Roll Subsystem:

$$
\begin{gathered}
{\left[\begin{array}{c}
\dot{x} \\
\ddot{x} \\
\dot{\phi} \\
\ddot{\phi}
\end{array}\right]=\underbrace{\left[\begin{array}{cccc}
0 & 1 & 0 & 0 \\
0 & 0 & -g & 0 \\
0 & 0 & 0 & 1 \\
0 & 0 & 0 & 0
\end{array}\right]}_{\mathbf{A}_{\text {roll }}}+\overbrace{\left[\begin{array}{c}
x \\
\dot{x} \\
\phi \\
\dot{\phi}
\end{array}\right]}^{\text {State }\left(\mathbf{x}_{\text {roll }}\right)}+\underbrace{\left[\begin{array}{c}
0 \\
0 \\
0 \\
\frac{l}{I_{x}}
\end{array}\right]}_{\mathbf{B}_{\text {roll }}} \overbrace{\left[\tau_{\phi}\right]}^{\text {Input }}} \\
y=\underbrace{\left[\begin{array}{llll}
0 & 1 & 0 & 0
\end{array}\right]}_{\mathbf{c}_{\text {roll }}} \mathbf{x}_{\text {roll }}
\end{gathered}
$$

III. $\mathcal{L}_{1}$ Adaptive Output Feedback Control

Fig. 3 shows the closed-loop $\mathcal{L}_{1}$ adaptive output feedback control structure proposed by [12]. The main purpose of adaptive controllers is the ability of conforming to any uncertainties which may arise in the dynamics. The QuadRotor UAV system equations presented in Section II can be expressed in the following form:

$$
y(s)=G(s)(u(s)+d(s)), \quad y(0)=0 .
$$

Where $u(t) \in \mathbb{R}$ is the input, $y(t) \in \mathbb{R}$ is the system output, $G(s)$ is the unknown transfer function of the system with the form $G(s)=c^{\top}(s \mathbb{I}-A)^{-1} B$ where $A, B$, and $c$ are 
taken from the systems (13)-(14) and (15)-(16); $d(s)$ is the Laplace transform of the time-varying uncertainties in the parameters of the system $d(t)=f(t, y(t))$ where $f$ is an unknown map, subject to Assumption 3 [30].

Assumption 3: There exists a constant $L_{d}>0$ such that the following inequality holds uniformly in $t \geq 0$ for all $y_{1}, y_{2}$ :

$$
\left|f\left(t, y_{1}\right)-f\left(t, y_{2}\right)\right| \leq L_{d}\left|y_{1}-y_{2}\right|
$$

Notice that $d(t)$ may depend on the system output $y$, and the upper bound of the growth rate of $d(t)$ with respect to $y$ is $L_{d}$. In this work, $d(t)$ does not depend on the system output $y(t)$, therefore the condition in (23) is satisfied automatically ¡30].

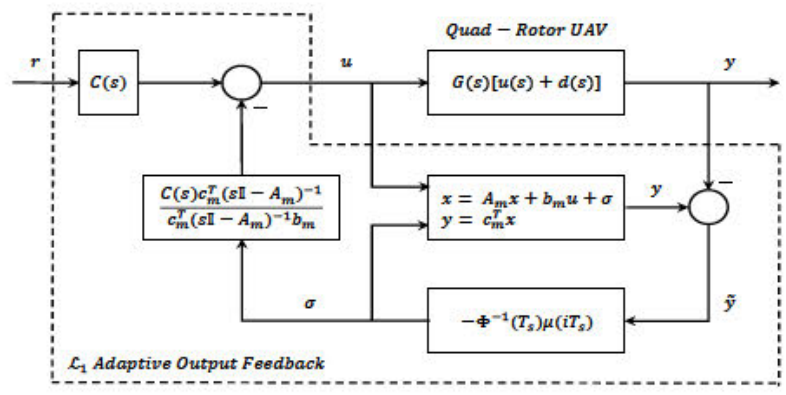

Fig. 3: $\mathcal{L}_{1}$ Adaptive Output Feedback Control Structure.

The objective, is to design an adaptive output feedback controller $u(t)$ such that the system output $y(t)$ tracks the given continuous reference input signal $r(t)$ even in the presence of uncertainties. This can be done by following a desired reference model, i.e. $y(s) \approx M(s) r(s)$; Where $M(s)$ is selected as a minimum-phase and stable transfer function. For developing this design objective, the system in (17) can be rewritten as:

$$
\begin{gathered}
y(s)=M(s)(u(s)+\sigma(s)) \\
\sigma(s)=((G(s)-M(s)) u(s)+G(s) d(s)) / M(s)
\end{gathered}
$$

where the uncertainties due to $G(s)$ and $d(s)$ are lumped into the signal $\sigma(s)$. Suppose $A_{m}, b_{m}$ and $c_{m}$ to be the minimal realization of $M(s)$. This means, $\left(A_{m}, b_{m}, c_{m}\right)$ is reachable and observable, with $A_{m}$ being Hurwitz. Therefore, the system in (18) can be rewritten as:

$$
\begin{gathered}
\dot{x}(t)=A_{m} x(t)+b_{m}(u(t)+\sigma(t)) \\
y(t)=c_{m}^{\top} x(t), \quad x(0)=x_{0}=0 .
\end{gathered}
$$

\section{A. Closed-loop Reference System}

Here, the closed-loop reference system that defines a realizable control objective for the $\mathcal{L}_{1}$ adaptive controller will be introduced. The reference system is given by:

$$
\begin{aligned}
& y_{r e f}(s)=M(s)\left(u_{r e f}(s)+\sigma_{r e f}(s)\right) \\
& \sigma_{r e f}(s)=\frac{\left((G(s)-M(s)) u_{r e f}(s)+G(s) d_{r e f}(s)\right)}{M(s)} \\
& u_{r e f}(s)=C(s)\left(r(s)-\sigma_{r e f}(s)\right)
\end{aligned}
$$

Where $d_{\text {ref }}(t)=f\left(t, y_{\text {ref }}(t)\right)$, and $C(s)$ is a low-pass filter of relative order $d_{r}$ with DC gain $C(0)=1$. In order for the reference system in (21) to be stable, the selection of $C(s)$ and $M(s)$ must ensure that

$$
H(s)=G(s) M(s) /(C(s) G(s)+(1-C(s)) M(s))
$$

is stable and

$$
\|N(s)\|_{\mathcal{L}_{1}} L_{d}<1,
$$

where $N(s)=H(s)(1-C(s))$. [4, Lemma 1]

\section{B. State Predictor}

Given the system in (20), and that $A_{m} \in \mathbb{R}^{n \times n}, b_{m} \in \mathbb{R}^{n}$ and $c_{m} \in \mathbb{R}^{n}$ are the minimal realization of $M(s)$. The state predictor is given by:

$$
\begin{aligned}
& \dot{\hat{x}}(t)=A_{m} \hat{x}(t)+b_{m} u(t)+\hat{\sigma}(t) \\
& \hat{y}(t)=c_{m}^{\top} \hat{x}(t) .
\end{aligned}
$$

where $\hat{\sigma}(t) \in \mathbb{R}^{n}$ is the vector of adaptive parameters. Note that while $\sigma(t) \in \mathbb{R}$ in (20), i.e. the unknown disturbance is matched, the uncertainty estimation in (24) is $\hat{\sigma}(t) \in \mathbb{R}^{n}$, i.e. the estimation of it is unmatched. This is a key step of the solution and the analysis in [6]. Taking the Laplace transform of (24), gives

$$
\hat{y}(s)=M(s) u(s)+c_{m}^{\top}\left(s \mathbb{I}-A_{m}\right)^{-1} \hat{\sigma}(s) .
$$

\section{Adaptation Law}

Since $A_{m}$ is Hurwitz, there exists $P=P^{\top}>0$ that satisfies the following algebraic Lyapunov equation:

$$
A_{m}^{\top} P+P A_{m}=-Q
$$

where $Q>0$. From the properties of $P$ it follows that there always exists a nonsingular $\sqrt{P}$, such that, $P=$ $\sqrt{P}^{\top} \sqrt{P}$. Given the vector $c_{m}^{\top}(\sqrt{P})^{-1}$, let $D$ be the $(n-$ 1) $\times n$-dimensional null-space of $c_{m}^{\top}(\sqrt{P})^{-1}$, i.e.

$$
D\left(c_{m}^{\top}(\sqrt{P})^{-1}\right)^{\top}=0
$$

and let

$$
\Lambda=\left[\begin{array}{c}
c_{m}^{\top} \\
D \sqrt{P}
\end{array}\right] \in \mathbb{R}^{n \times n} .
$$

Letting $\tilde{y}(t)=\hat{y}(t)-y(t)$, the update law for $\hat{\sigma}(t)$ is defined via the sampling time $T>0$ which defines the sampling rate of the available CPU:

$$
\hat{\sigma}(i T)=-\Phi^{-1}(T) \mu(i T), \quad i=1,2, \cdots,
$$

where

$$
\Phi(T)=\int_{0}^{T} e^{\Lambda A_{m} \Lambda^{-1}(T-\tau)} \Lambda d \tau
$$

and

$$
\mu(i T)=e^{\Lambda A_{m} \Lambda^{-1} T} \mathbf{1}_{1} \tilde{y}(i T), \quad i=1,2, \cdots
$$

With $1_{1}$ a basis vector in the space $\mathbb{R}^{n}$. 


\section{Control Law}

The control law is defined via the output of the low-pass filter:

$$
u(s)=C(s) r(s)-\frac{C(s)}{M(s)} c_{m}^{\top}\left(s \mathbb{I}-A_{m}\right)^{-1} \hat{\sigma}(s) .
$$

The complete $\mathcal{L}_{1}$ adaptive controller consists of the state predictor in (24), the adaptation law in (29) and the control law in (32), subject to the $\mathcal{L}_{1}$-gain upper bound in (23). The authors recommend consulting [6] for complete proofs and analysis of the $\mathcal{L}_{1}$ adaptive output feedback controller.

\section{Simulation Results}

The $\mathcal{L}_{1}$ adaptive output feedback control approach has been implemented for velocity control of a Quad-Rotor UAV in the $x$ and $y$ (forward and lateral) directions. Two flight scenarios are then tested to verify the performance of the $\mathcal{L}_{1}$ control design and compare these results to the performance of a basic LQR controller. Section IV-B presents the outputs of the system without disturbances, whereas, Section IV-C introduces an added disturbance on the $x$ and $y$ directions.

\section{A. Simulation Setup}

The dynamic model of the system (1)-(6), presented in Section II, was implemented to test the results of the controller designs on a full non-linear simulation of the QuadRotor UAV. This simulation was performed in the Simulink environment of MATLAB 2016a and the parameters of the system are detailed in Table I. These parameters have been chosen to match, as close as possible, the parameters of the Quad-Rotor UAV to be used in Section V for real flight tests.

TABLE I: Quad-Rotor Simulation Model Parameters

\begin{tabular}{c||c||c||c}
\hline \multicolumn{1}{c||}{ Parameter } & Description & Value & Units \\
\hline \hline$l$ & Distance from the rotors to the center of mass & 0.18 & {$[\mathrm{~m}]$} \\
\hline$m$ & Mass of the system & 0.472 & {$[\mathrm{~kg}]$} \\
\hline$T_{s}$ & Sampling Time & 0.0001 & {$[\mathrm{~s}]$} \\
\hline$g$ & Gravity Constant & 9.81 & {$\left[\mathrm{~m} \cdot \mathrm{s}^{-2}\right]$} \\
\hline$I_{x}$ & Moment of inertia about $x$-axis & 0.01152 & {$\left[\mathrm{~kg} \cdot \mathrm{m}^{2}\right]$} \\
\hline$I_{y}$ & Moment of inertia about $y$-axis & 0.01152 & {$\left[\mathrm{~kg} \cdot \mathrm{m}^{2}\right]$} \\
\hline$I_{z}$ & Moment of inertia about $z$-axis & 0.0218 & {$\left[\mathrm{~kg} \cdot \mathrm{m}^{2}\right]$} \\
\hline
\end{tabular}

The tuning of $C(s)$ and $M(s)$ transfer functions from the closed loop reference system in (21), which we omit here, was based on the work detailed in [30], where an $\mathcal{L}_{1}$ controller for a missile autopilot was developed. The $C(s)$ and $M(s)$ transfer functions which were able to achieve the best performance for velocity control in the $x$ direction, are shown in (33) and (34) respectively. $C(s)$ was chosen as a second order relative degree 2 transfer function where $C(0)=1$ and $M(s)$ to be a fourth order relative degree 1 transfer function.

$$
\begin{gathered}
C(s)=\frac{1.504 e 04}{s^{2}+40 e 03 s+1.504 e 04} \\
M(s)=\frac{(s+63.91)\left(s^{2}+51.09 s+2310\right)}{(s+4 e 04)(s+3.803)\left(s^{2}+5.4 s+25.67\right)}
\end{gathered}
$$

\section{B. Simulation results (No Wind Gust Disturbance)}

The output velocities in the $x$ and $y$ (forward and lateral) directions of the system, with the $\mathcal{L}_{1}$ Adaptive Control method and the LQR, are shown in Figs. 4(a) and 4(b) respectively. As can be seen, both controllers are able to track the step reference input $r(t)=1 \mathrm{~m} \cdot \mathrm{s}^{-1}$ at $t=1$ $\mathrm{s}$ with satisfactory performance. They exhibit quite similar output responses and also have a reasonable settling time. Even though the LQR controller performance in these tests is acceptable, it will later be demonstrated that in the presence of wind gust disturbances the response is significantly different.

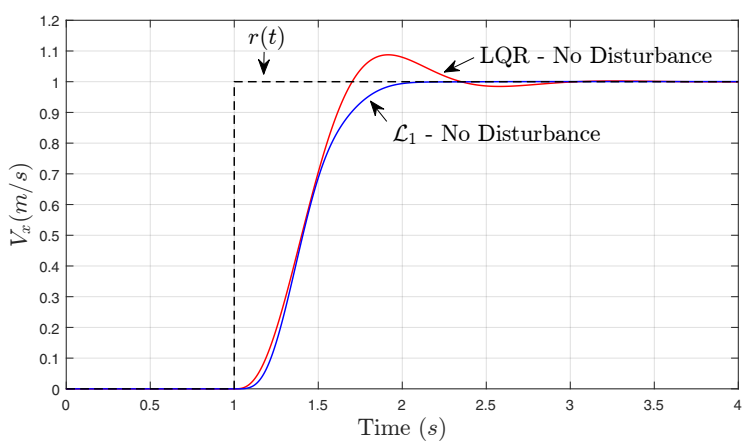

(a)

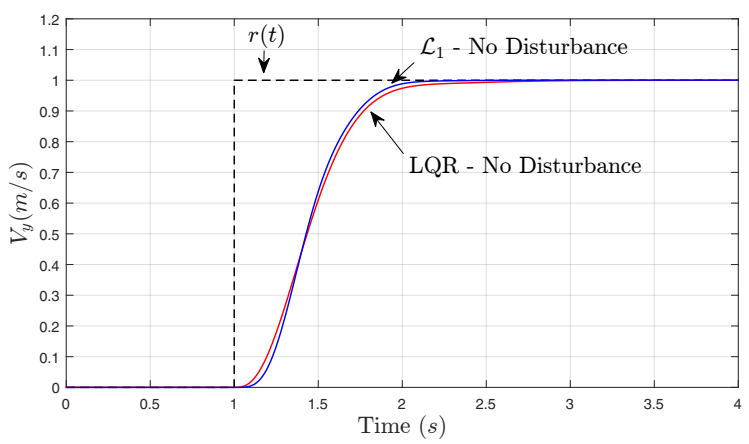

(b)

Fig. 4: Velocity control simulation results of a step reference input for a non-disturbed system using $\mathcal{L}_{1}$ and LQR control. (a) Velocity output in the $x$ (forward) direction. (b) Velocity output in the $y$ (lateral) direction.

\section{Simulation Results (Wind Gust Disturbance)}

For the second scenario in a simulated environment, both control methods were tested to validate the performance of each controller when wind gusts affect the dynamics of the system. The wind gust disturbances applied to the system in simulation can be seen in Figs. 5(a) and 5(c). Wind gust profiles were generated using random data, with gusts reaching up to $4 \mathrm{~m} \cdot \mathrm{s}^{-1}$ in the positive $x$ direction and $2 \mathrm{~m} \cdot \mathrm{s}^{-1}$ in the positive $y$ direction which were applied throughout the whole simulation. These values were chosen with high variance $\left(\mathrm{e} . \mathrm{g} .0 \mathrm{~m} \cdot \mathrm{s}^{-1}\right.$ to $3 \mathrm{~m} \cdot \mathrm{s}^{-1}$ in less than $1 \mathrm{~s}$ ) to test in simulation extreme cases and validate the control methods before performing the real flight tests shown in Section V. 


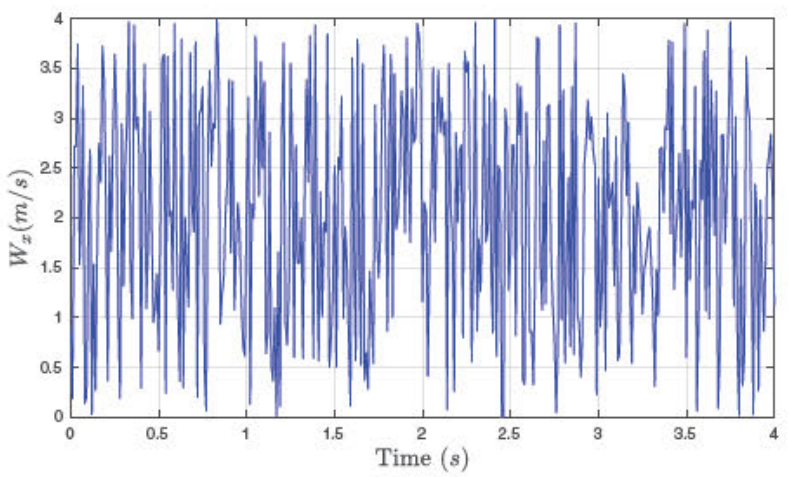

(a)

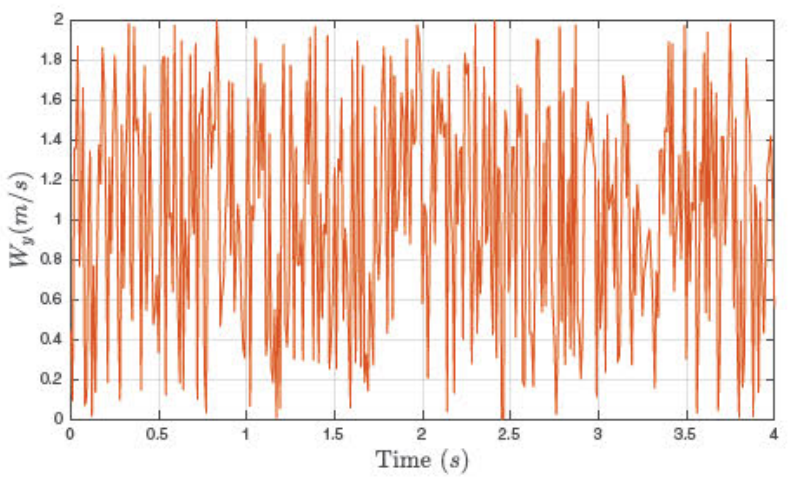

(c)

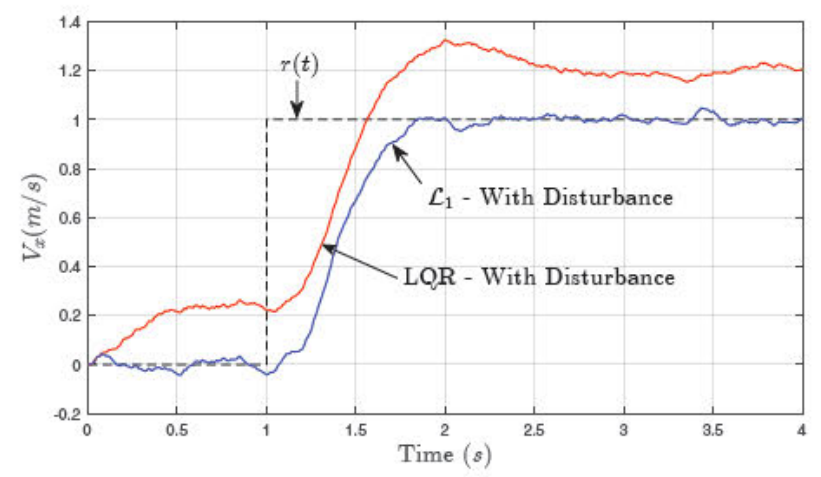

(b)

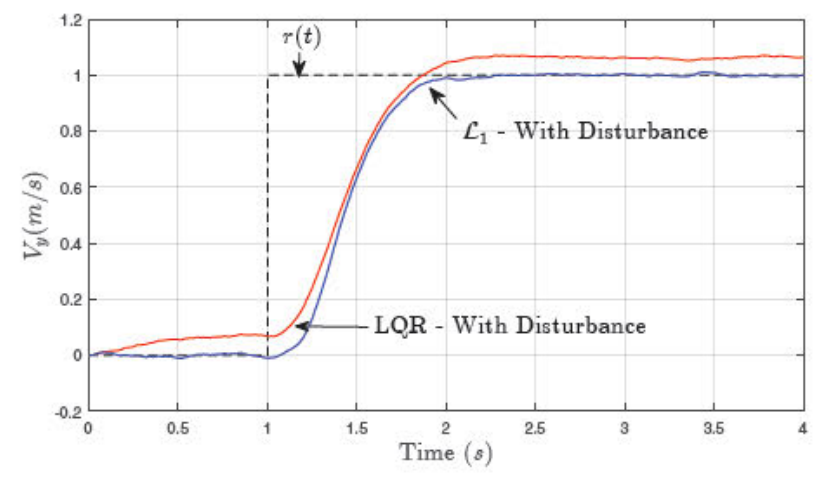

(d)

Fig. 5: Velocity control simulation results. A step reference input was applied to the system with external disturbances and controlled using $\mathcal{L}_{1}$ and LQR control. (a) Wind profile added in the $x$ direction. (b) Velocity output in the $x$ (forward) direction. (c) Wind profile added in the $y$ direction. (d) Velocity output in the $y$ (lateral) direction.

In Table II, the evaluation metrics for the wind gust simulation test are presented, these are; percentage overshoot $(P O)$, rise time $\left(t_{r}\right)$, steady-state velocity $(V(s s))$ and the offset from the desired reference to the steady-state value. Figs. 5(b) and 5(d) show the output velocity, in $x$ and $y$ direction of the system, for a step reference of $r(t)=1$ $\mathrm{m} \cdot \mathrm{s}^{-1}$ at $t=1 \mathrm{~s}$ using the $\mathcal{L}_{1}$ adaptive and LQR controllers. From these results, it is evident that both control methods are capable of stabilizing the system without notable oscillations, even in the presence of added wind gust disturbances in both directions.

As can be seen in Table II, the $\mathcal{L}_{1}$ adaptive controller has considerably better performance than the LQR. The latter method is shown to be unable to maintain the desired reference in steady-state by demonstrating an offset of 0.185 $\mathrm{m} \cdot \mathrm{s}^{-1}$ from the reference velocity in $x$ and $0.057 \mathrm{~m} \cdot \mathrm{s}^{-1}$ in $y$ for different wind speeds along each axis. However, in the presence of the same wind gusts where the LQR method had an offset of $0.185 \mathrm{~m} \cdot \mathrm{s}^{-1}$, the $\mathcal{L}_{1}$ controller presented only an offset of $-0.0142 \mathrm{~m} \cdot \mathrm{s}^{-1}$ from the desired reference velocity. Additionally, the $\mathcal{L}_{1}$ controller achieved a steadystate value of $0.986 \mathrm{~m} \cdot \mathrm{s}^{-1}$ in $x$ and $0.993 \mathrm{~m} \cdot \mathrm{s}^{-1}$ in $y$, which is highly desirable. This validates the robustness of this adaptive control method and provides a suitable approach for real flight tests.
TABLE II: Wind Disturbance Simulation Evaluation

\begin{tabular}{c|c|c||c|c|c}
\cline { 2 - 6 } & \multicolumn{2}{|c||}{$V_{\boldsymbol{x}}$} & \multicolumn{2}{c|}{$V_{\boldsymbol{y}}$} & \\
\hline Metrics & LQR & $\mathcal{L}_{1}$ & LQR & $\mathcal{L}_{1}$ & Units \\
\hline \hline$P O$ & 14.58 & $\mathbf{2 . 5 9 8}$ & $\mathbf{0 . 5 3 8}$ & 1.077 & {$[\%]$} \\
\hline$t_{r}$ & $\mathbf{0 . 4 1 9}$ & 0.450 & 0.597 & $\mathbf{0 . 5 0 3}$ & {$[\mathrm{s}]$} \\
\hline$V(s s)$ & 1.185 & $\mathbf{0 . 9 8 6}$ & 1.057 & $\mathbf{0 . 9 9 3}$ & {$\left[\mathrm{m} \cdot \mathrm{s}^{-1}\right]$} \\
\hline off set & 0.1850 & $\mathbf{- 0 . 0 1 4 2}$ & 0.0570 & $\mathbf{- 0 . 0 0 7 3}$ & {$\left[\mathrm{m} \cdot \mathrm{s}^{-1}\right]$} \\
\hline
\end{tabular}

\section{Preliminary Experimental Results}

Simulation results show that the adaptive $\mathcal{L}_{1}$ control design is a feasible solution for Quad-Rotor UAV flights in the presence of wind gusts. Nevertheless, real flight test are performed to validate these results. The values for the state predictor (24), adaptation law (29) and control law (32) of the $\mathcal{L}_{1}$ adaptive control design in the experimental tests, were chosen to be the same as the ones used in simulation. This was done in order to test the adaptation capability of the controller towards changes in the parameters between the real platform and the dynamic model used for design. The experimental tests performed can be seen in the following video: https://vimeo.com/205753712. 

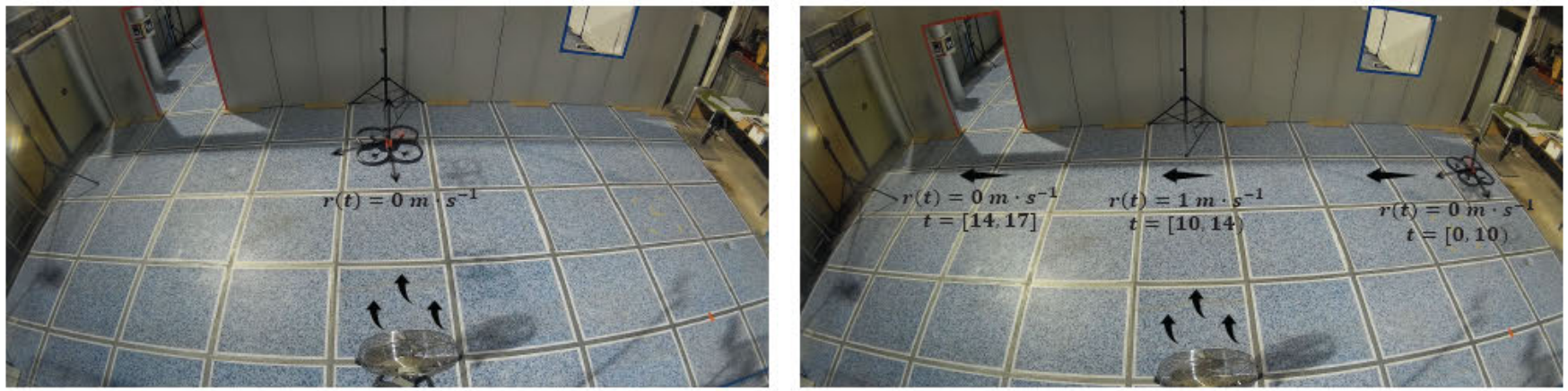

Fig. 6: Indoor experimental setup used for real flight tests. The location was in an $9 \mathrm{~m} \mathrm{x} 5 \mathrm{~m}$ indoor arena with no motion capture system. A fan with a maximum airflow of $5 \mathrm{~m} \cdot \mathrm{s}^{-1}$ was used as a disturbance generator. (Left) Quad-Rotor placement for wind and no wind hover experimental tests. (Right) Quad-Rotor placement for step response experimental test.

\section{A. Experimental Setup}

The Quad-Rotor UAV platform chosen to test the control methods was the Parrot Ar.Drone 2.0. This lightweight QuadRotor is extensively used by researchers given its ease of use and low-cost; which allows for rapid testing of algorithms, before implementing on bigger platforms. It is controlled by a ground-station PC running Ubuntu 16.04 and the Robot Operating System (ROS) Kinetic. In order to send commands to the Ar.Drone 2.0, the open-source framework Aerostack ${ }^{12}$ was used [21] [22]. This framework includes components for control, state-estimation, mission planning, etc.

The experimental setup can be seen in Fig. 6. The QuadRotor was flown in a space of $9 \mathrm{~m} \mathrm{x} 5 \mathrm{~m}$ of an indoor flying arena at the Technical University of Madrid Center for Automation and Robotics. Although motion capture systems, such as Optitrack ${ }^{3}$, can offer precise measurements to aid in the testing of control algorithms, the performance achieved is not realistic when compared to flying in unknown environments where you have to rely on state-estimation algorithms to have quality feedback measurements. Therefore, the controller was tuned and tested with the non-ideal measurements from the on-board sensors.

However, Aerostack provides state-estimation algorithms that have been extensively validated with the Ar.Drone 2.0 platform [23]. Additionally, Aerostack offers a baseline PID velocity controller which is already implemented and tuned in the framework, this is used for comparison with the performance of the $\mathcal{L}_{1}$ controller in real flights. A fan is placed at a distance of $1.5 \mathrm{~m}$ in the positive $y$ direction blowing towards the negative $y$ direction orthogonal to the $x$ axis. This fan is used as a wind gust disturbance generator and was measured to have non-uniform wind speeds from the exterior to the center of the fan and reaching up to $5 \mathrm{~m} \cdot \mathrm{s}^{-1}$. These speeds were thought to be sufficient for testing on a small platform such as the Ar.Drone 2.0.

Two scenarios have been contemplated to test the response of a real Quad-Rotor UAV platform; first, a simple hover test, where $r(t)=0 \mathrm{~m} \cdot \mathrm{s}^{-1}$, with and without added wind gust disturbances (Section V-B and Section V-C); and second, a

\footnotetext{
${ }^{1}$ Aerostack webpage: http://www. aerostack.org/

${ }^{2}$ GitHub :https://github.com/Vision 4 UAV/Aerostack

${ }^{3}$ Optitrack Webpage: http://www.optitrack.com/
}

velocity step reference of $r(t)=1 \mathrm{~m} \cdot \mathrm{s}^{-1}$ is given for $4 \mathrm{~s}$ in the $x$ direction where it will encounter the gust disturbance in the $y$ direction mid-flight (Section V-D).

\section{B. Hover Results (No Wind Gust Disturbance)}

The purpose of this initial test was to validate the $\mathcal{L}_{1}$ control response and its ability to maintain a real QuadRotor platform stabilized in a simple scenario, without wind disturbances. Fig. 7 shows the output velocities in $x$ and $y$ direction for a duration of $20 \mathrm{~s}$. Test results for Root Mean Square error (RMSe), maximum velocity $\left(V_{\max }\right)$ and minimum velocity $\left(V_{\min }\right)$ are shown in Table III. These results reflect the $\mathcal{L}_{1}$ controller performance during hover. The $\mathcal{L}_{1}$ control approach is able to maintain the output velocities with minimal deviation from the reference producing satisfactory RMSe values of $0.0099 \mathrm{~m} \cdot \mathrm{s}^{-1}$ for the $x$ direction and 0.0230 $\mathrm{m} \cdot \mathrm{s}^{-1}$ for the $y$ direction.

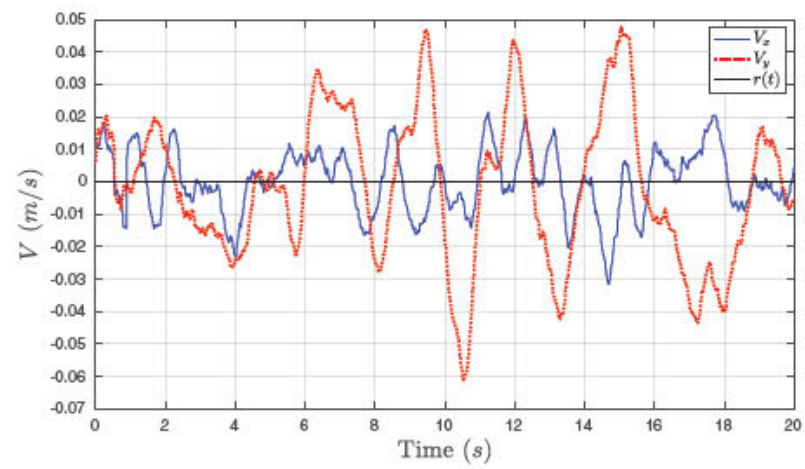

Fig. 7: Output velocities in $x$ and $y$ directions for a $20 \mathrm{~s}$ undisturbed hover test with the $\mathcal{L}_{1}$ control method.

TABLE III: Hover Flight Results - No Wind Disturbance

\begin{tabular}{c|c|c|c}
\hline Metrics & $V_{x}$ & $V_{y}$ & Units \\
\hline RMSe & 0.0099 & 0.0230 & {$\left[\mathrm{~m} \cdot \mathrm{s}^{-1}\right]$} \\
\hline$V_{\max }$ & 0.0212 & 0.0476 & {$\left[\mathrm{~m} \cdot \mathrm{s}^{-1}\right]$} \\
\hline$V_{\text {min }}$ & -0.0315 & -0.0613 & {$\left[\mathrm{~m} \cdot \mathrm{s}^{-1}\right]$} \\
\hline
\end{tabular}




\section{Hover Results (With Wind Gust Disturbance)}

In Fig. 8, the results for the hover test with added wind gust disturbances can be seen. For this test, as shown in Fig. 6 (Left), the Quad-Rotor is placed directly in front of the fan whose airflow is pointed towards the negative $y$ direction of the UAV. The system is given reference input of $r(t)=0$ $\mathrm{m} \cdot \mathrm{s}^{-1}$ in both $x$ and $y$ directions for a duration of $37 \mathrm{~s}$.

Figs. 8(a) and 8(b) show the output velocities in the $x$ and $y$ directions, respectively, controlled by the $\mathcal{L}_{1}$ and the PID. Throughout the first $7 \mathrm{~s}$ of hover, both the $\mathcal{L}_{1}$ controller (as was expected from the no wind flight performed in Section V-B) and the PID controller are capable of maintaining the desired reference value and perform perfectly when no wind is applied, exhibiting negligible errors. After stabilizing for $7 \mathrm{~s}$, the fans airflow was turned on for the remainder of the test. Evidently, the effects of the wind disturbances alters the performance of both controllers. Nonetheless, the $\mathcal{L}_{1}$ controller outperforms the PID by stabilizing the Quad-Rotor with less drift from the desired velocity reference.

In Table IV, the evaluation metrics for the hover flight with wind disturbance are detailed. As can be seen, in the direction of the wind disturbance, the PID controller reaches a maximum velocity of $0.3452 \mathrm{~m} \cdot \mathrm{s}^{-1}$ which is $56.90 \%$ higher than the $\mathcal{L}_{1}$ controllers maximum velocity of 0.22 $\mathrm{m} \cdot \mathrm{s}^{-1}$. In general the PID controller achieves RMSe values of $0.0529 \mathrm{~m} \cdot \mathrm{s}^{-1}$ and $0.1279 \mathrm{~m} \cdot \mathrm{s}^{-1}$ for velocities in the $x$ and $y$ directions respectively, more than $30 \%$ higher than those of the $\mathcal{L}_{1}$ controller which is able to maintain desirable RMSe values of $0.0404 \mathrm{~m} \cdot \mathrm{s}^{-1}$ and $0.0924 \mathrm{~m} \cdot \mathrm{s}^{-1}$ in the same directions.

TABLE IV: Hover Flight Results - With Wind Disturbance

\begin{tabular}{c|c|c||c|c|c}
\cline { 2 - 5 } & \multicolumn{2}{|c|}{$V_{\boldsymbol{x}}$} & \multicolumn{2}{c|}{$V_{\boldsymbol{y}}$} & \\
\hline Metrics & $\mathcal{L}_{1}$ & PID & $\mathcal{L}_{1}$ & PID & Units \\
\hline \hline RMSe & $\mathbf{0 . 0 4 0 4}$ & 0.0529 & $\mathbf{0 . 0 9 2 4}$ & 0.1279 & {$\left[\mathrm{~m} \cdot \mathrm{s}^{-1}\right]$} \\
\hline$V_{\max }$ & $\mathbf{0 . 1 8 2 4}$ & 0.1995 & $\mathbf{0 . 2 2 0 0}$ & 0.3452 & {$\left[\mathrm{~m} \cdot \mathrm{s}^{-1}\right]$} \\
\hline$V_{\min }$ & $\mathbf{- 0 . 1 6 0 2}$ & -0.1624 & $\mathbf{- 0 . 3 7 3 3}$ & -0.3939 & {$\left[\mathrm{~m} \cdot \mathrm{s}^{-1}\right]$} \\
\hline
\end{tabular}

\section{Step Response Results (With Wind Gust Disturbance)}

Considering that the results in the hover tests were acceptable, the next flight test was conducted to measure the performance of both control methods for a step reference input. Fig. 6 (Right) shows the general layout for this experimental test. The Quad-Rotor was put on hover in its initial position for a duration of $10 \mathrm{~s}$, afterwards, a step velocity reference signal of $r(t)=1 \mathrm{~m} \cdot \mathrm{s}^{-1}$ in the $x$ direction is sent for a duration of $4 \mathrm{~s}$, before returning to hover. Throughout the whole test, the reference signal in the $y$ direction is maintained at $r(t)=0 \mathrm{~m} \cdot \mathrm{s}^{-1}$.

Similar to the previous wind disturbance tests the fans airflow is pointed towards the negative $y$ direction of the $\mathrm{UAV}$, this means that the biggest effect of the wind airflow will reflect on the velocity in the $y$ direction of the QuadRotor.

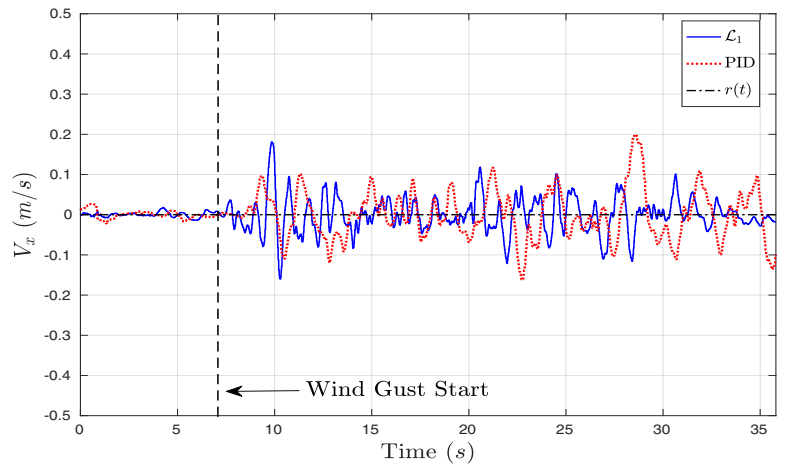

(a)

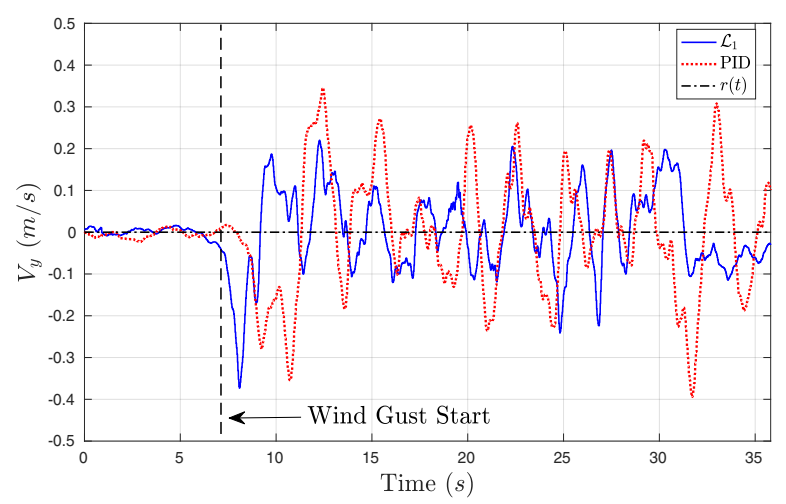

(b)

Fig. 8: Output Velocities of Quad-Rotor during the hover experimental test with wind gust disturbance added at $t=7$ s. (a) Velocity output in the $x$ direction. (b) Velocity output in the $y$ direction.

As shown in the plots in Fig. 9, the Quad-Rotor enters the fans airflow at approximately $t=12.38 \mathrm{~s}$ and exits at $t=$ 13.7 s. Fig. 9(b) presents the output velocity of the system in the $y$ direction for the step response test controlled with the $\mathcal{L}_{1}$ and PID controllers. It is clear that the $\mathcal{L}_{1}$ controller is able to compensate for the wind disturbance, at $t=12.38$ $\mathrm{s}$, much faster and with less error than the PID controller. After leaving the wind gust disturbance, at $t=13.7 \mathrm{~s}$, the $\mathcal{L}_{1}$ controller also stabilizes faster back to the desired reference. This is reflected in the $V_{y}$ columns of Table $\mathrm{V}$, where the PID generates $67.28 \%$ overshoot, while the $\mathcal{L}_{1}$ controller generates only $30.22 \%$. The $\mathcal{L}_{1}$ controller also achieves a RMSe value of $0.1126 \mathrm{~m} \cdot \mathrm{s}^{-1}$, which is $39.17 \%$ lower than the RMSe with the PID velocity controller in the $y$ direction.

In Fig. 9(a) the output velocity of the system in the $x$ direction, where the step reference has been sent, can be seen. The response of the PID is noticeably faster with a $t_{r}$ of $0.7615 \mathrm{~s}$, almost twice as fast as that of the $\mathcal{L}_{1}$ controller. However, for the step reference command, the $\mathcal{L}_{1}$ controllers performance yields much more desirable values where $P O=2.3081 \%, V(s s)=0.9849 \mathrm{~m} \cdot \mathrm{s}^{-1}$ and RMSe $=0.2696 \mathrm{~m} \cdot \mathrm{s}^{-1}$. 


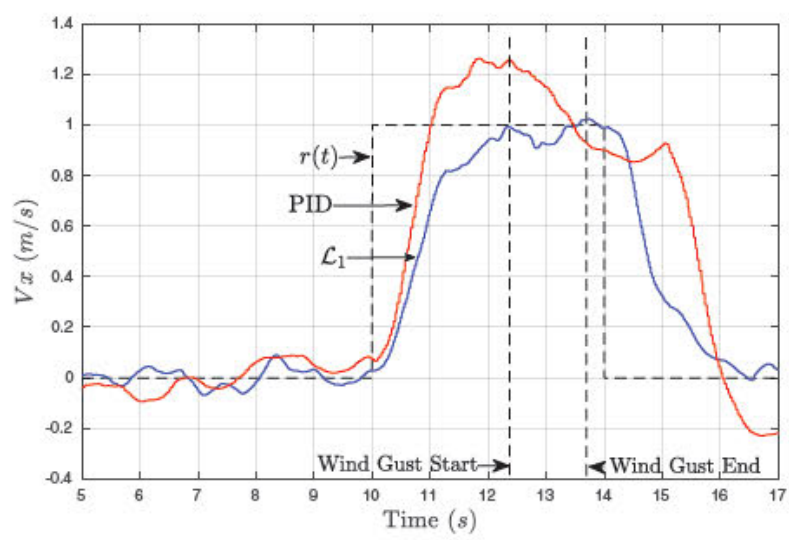

(a)

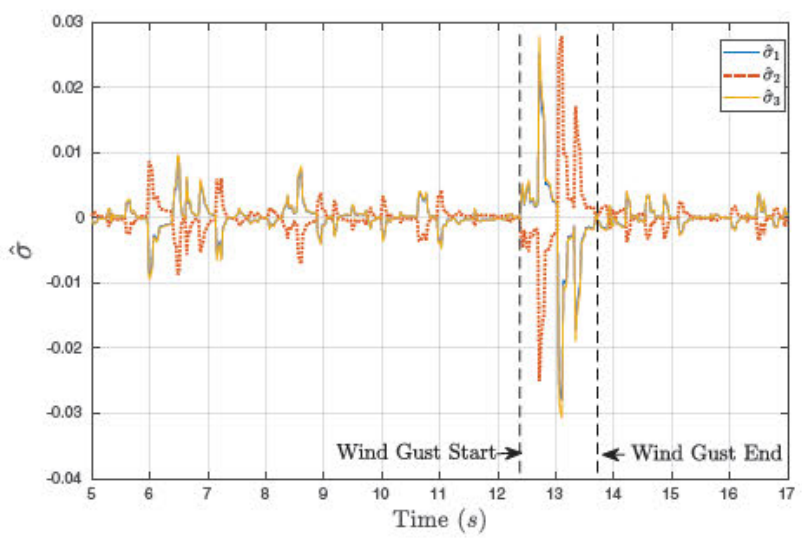

(c)

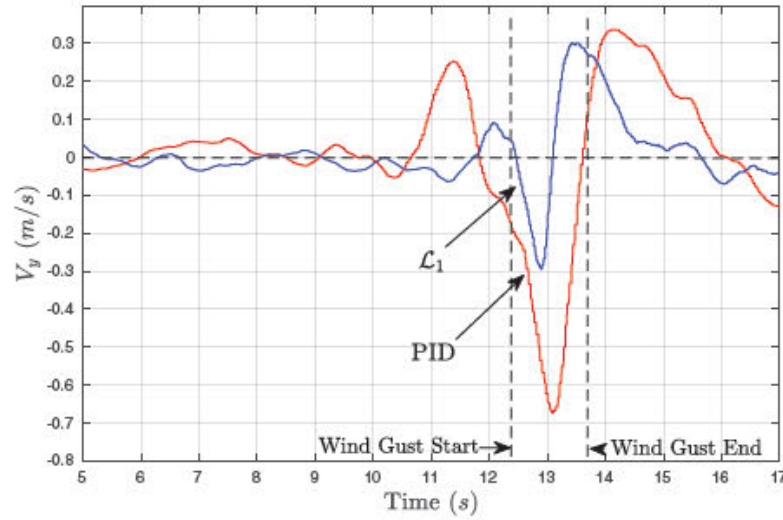

(b)

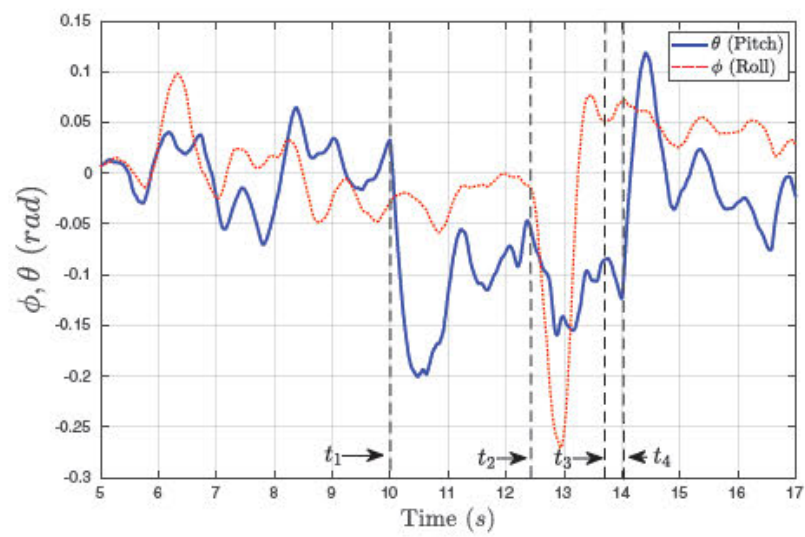

(d)

Fig. 9: Output velocities of a Quad-Rotor during the step reference experimental test with wind gust disturbance added at $t=12.38 \mathrm{~s}$ and stopped at $t=13.7 \mathrm{~s}$. (a) Velocity output in the $x$ direction. (b) Velocity output in the $y$ direction. (c) Adaptation parameters $(\hat{\sigma})$ in the $y$ direction. (d) Control signal of the $\mathcal{L}_{1}$ control method for $x$ and $y$ direction. $\left(t_{1}\right)$ Step reference sent, $\left(t_{2}\right)$ wind gust disturbance started, $\left(t_{3}\right)$ wind gust disturbance stopped, $\left(t_{4}\right) r(t)=0 \mathrm{~m} \cdot \mathrm{s}^{-1}$ reference sent.

Notice that when comparing the output response signal of the system controlled with the $\mathcal{L}_{1}$ controller shown in Fig. 9(a) with the response generated by the simulation in Section IV-C, the $\mathcal{L}_{1}$ controller is capable of generating a response very similar to the one shown in Fig. 5(b) where in almost $2 \mathrm{~s}$ the output is able to reach the desired reference with minimal deviation.

As was mentioned earlier, one of the main strengths of the $\mathcal{L}_{1}$ adaptive control method is that it guarantees a low-frequency control signal. This can be seen in Fig. 9(d) where the $\mathcal{L}_{1}$ controller generates a smooth control signal even in the presence of external disturbances without high-frequency oscillations, which was a drawback of other adaptive control methods. Another key aspect of the $\mathcal{L}_{1}$ control is the adaptation parameters (29). Fig. 9(c) shows the adaptive parameters of the velocity controller in the $y$ direction for the step response test. It can be seen that the adaptive parameters suddenly change in an attempt to cancel out the external disturbances when entering the airflow at $t=12.38 \mathrm{~s}$, while remaining within reasonable bounds. This capability of fast adaptation while maintaining robustness of the closed-loop system is the main difference between $\mathcal{L}_{1}$ adaptive control and other MRAC methods.

TABLE V: Step Reference Results - With Wind Disturbance

\begin{tabular}{c|c|c||c|c|c}
\cline { 2 - 5 } & \multicolumn{2}{c||}{$V_{\boldsymbol{x}}$} & \multicolumn{2}{c|}{$V_{\boldsymbol{y}}$} & \multicolumn{1}{c}{} \\
\hline Metrics & $\mathcal{L}_{1}$ & PID & $\mathcal{L}_{1}$ & PID & Units \\
\hline \hline RMSe & $\mathbf{0 . 2 6 9 6}$ & 0.3199 & $\mathbf{0 . 1 1 2 6}$ & 0.1851 & {$\left[\mathrm{~m} \cdot \mathrm{s}^{-1}\right]$} \\
\hline$P O$ & $\mathbf{2 . 3 0 8 1}$ & 26.208 & $\mathbf{3 0 . 2 2 0}$ & 67.280 & {$[\%]$} \\
\hline$t_{r}$ & 1.5476 & $\mathbf{0 . 7 6 1 5}$ & $N / A$ & $N / A$ & {$[s]$} \\
\hline$V(s s)$ & $\mathbf{0 . 9 8 4 9}$ & 0.8967 & $N / A$ & $N / A$ & {$\left[\mathrm{~m} \cdot \mathrm{s}^{-1}\right]$} \\
\hline$V_{\max }$ & $\mathbf{1 . 0 2 3}$ & 1.262 & $\mathbf{0 . 3 0 2 2}$ & 0.3364 & {$\left[\mathrm{~m} \cdot \mathrm{s}^{-1}\right]$} \\
\hline$V_{\min }$ & $\mathbf{- 0 . 0 6 7 6}$ & -0.2293 & $\mathbf{- 0 . 2 9 3 8}$ & -0.6728 & {$\left[\mathrm{~m} \cdot \mathrm{s}^{-1}\right]$} \\
\hline
\end{tabular}




\section{COnClusions And Future Work}

In this paper an $\mathcal{L}_{1}$ adaptive output feedback velocity controller was developed for wind gust rejection in a QuadRotor UAV, during high wind inspection applications. The preliminary results shown, confirm the robustness of the adaptive control method and prove that this approach is a viable solution for compensating wind gust disturbances in wind turbine inspection tasks. Several tests have been performed in simulation and in real flights to evaluate the performance and robustness of the $\mathcal{L}_{1}$ control method in a Quad-Rotor UAV which can be seen in video.

It is demonstrated, that the performance of the $\mathcal{L}_{1}$ controller exceeds the performance of classical control strategies such as LQR in simulation and PID in real flights. Overall, the response of the $\mathcal{L}_{1}$ controller meets the goals of tracking the reference signal smoothly while adapting to unknown disturbances and maintaining minimal steady-state error with low frequency control signals. The adaptability of the $\mathcal{L}_{1}$ controller was established with the use of the simulation values for the $\mathcal{L}_{1}$ design in the real system and the robustness was seen in the real flight tests where the flight of a QuadRotor UAV was satisfactory even when disturbed by a fans wake.

Future work includes, implementing the LQR controller used in simulation for testing in real flights, the outer loop position controller in order to test the wind gust resistance while maintaining a way-point instead of a velocity reference. Additionally, the $\mathcal{L}_{1}$ control method will be compared to other control methods such as Geometric Control or Model Predictive Control.

\section{REFERENCES}

[1] Brian D. O. Anderson. Failures of adaptive control theory and their resolution. Commun. Inf. Syst., 05(1):1-20, 2005.

[2] J. X. J. Bannwarth, Z. J. Chen, K. A. Stol, and B. A. MacDonald. Disturbance accomodation control for wind rejection of a quadcopter. In 2016 International Conference on Unmanned Aircraft Systems (ICUAS), pages 695-701, June 2016.

[3] S. Bouabdallah, P. Murrieri, and R. Siegwart. Design and control of an indoor micro quadrotor. In Robotics and Automation, 2004. Proceedings. ICRA '04. 2004 IEEE International Conference on, volume 5, pages 4393-4398 Vol.5, April 2004.

[4] C. Cao and N. Hovakimyan. L1 adaptive output feedback controller to systems of unknown dimension. In 2007 American Control Conference, pages 1191-1196, July 2007.

[5] C. Cao and N. Hovakimyan. L1 adaptive controller for systems with unknown time-varying parameters and disturbances in the presence of non-zero trajectory initialization error. International Journal of Control, 81(7):1147-1161, 2008.

[6] C. Cao and N. Hovakimyan. L1 adaptive output feedback controller for non strictly positive real reference systems with applications to aerospace examples. In In Proc. AIAA Guidance, Navigation, and Control Conf, 2008.

[7] Chengyu Cao and N. Hovakimyan. Design and analysis of a novel 11 adaptive controller, part i: Control signal and asymptotic stability. In 2006 American Control Conference, pages 3397-3402, June 2006.

[8] I. Golightly and D. Jones. Visual control of an unmanned aerial vehicle for power line inspection. In ICAR '05. Proceedings., 12th International Conference on Advanced Robotics, 2005., pages 288295, July 2005.

[9] John Hauser, Shankar Sastry, and George Meyer. Nonlinear control design for slightly non-minimum phase systems: Application to v/stol aircraft. Automatica, 28(4):665-679, 1992.
[10] Gabriel M. Hoffmann, Haomiao Huang, Steven L. Wasl, and Er Claire J. Tomlin. Quadrotor helicopter flight dynamics and control: Theory and experiment. In In Proc. of the AIAA Guidance, Navigation, and Control Conference, 2007.

[11] Gabriel M. Hoffmann, Steven L. Wasl, and Claire J. Tomlin. Quadrotor helicopter trajectory tracking control. In In Proc. AIAA Guidance, Navigation, and Control Conf, 2008.

[12] Naira Hovakimyan and Chengyu Cao. L1 Adaptive Control Theory: Guaranteed Robustness with Fast Adaptation. Society for Industrial and Applied Mathematics, Philadelphia, PA, USA, 2010.

[13] Y. Jung, S. Cho, and D. H. Shim. A trajectory-tracking controller design using 11 adaptive control for multi-rotor uavs. In 2015 International Conference on Unmanned Aircraft Systems (ICUAS), pages 132-138, June 2015.

[14] Daewon Lee, H Jin Kim, and Shankar Sastry. Feedback linearization vs. adaptive sliding mode control for a quadrotor helicopter. International Journal of control, Automation and systems, 7(3):419-428, 2009.

[15] N. Liu, Z. Cai, and Y. Wang. An 11 adaptive roll and pitch angle controller for quadrotors. In 2016 Chinese Control and Decision Conference (CCDC), pages 2473-2478, May 2016.

[16] Srinath Mallikarjunan, Bill Nesbitt, Evgeny Kharisov, Enric Xargay, Naira Hovakimyan, and Chengyu Cao. L1 Adaptive controller for attitude control of multirotors. 2012

[17] C. Martinez, C. Sampedro, A. Chauhan, and P. Campoy. Towards autonomous detection and tracking of electric towers for aerial power line inspection. In Unmanned Aircraft Systems (ICUAS), 2014 International Conference on, pages 284-295, May 2014.

[18] Daniel Mellinger, Nathan Michael, and Vijay Kumar. Trajectory generation and control for precise aggressive maneuvers with quadrotors The International Journal of Robotics Research, 31(5):664-674, 2012

[19] Kumpati S Narendra and Snehasis Mukhopadhyay. Adaptive control using neural networks and approximate models. IEEE Transactions on neural networks, 8(3):475-485, 1997.

[20] Vijay V. Patel, Chengyu Cao, Naira Hovakimyan, Kevin A. Wise, and Eugene Lavretsky. L1 adaptive controller for tailless unstable aircraft in the presence of unknown actuator failures. International Journal of Control, 82(4):705-720, 2009

[21] J. L. Sanchez-Lopez, R. A. Suárez Fernández, H. Bavle, C. Sampedro, M. Molina, J. Pestana, and P. Campoy. Aerostack: An architecture and open-source software framework for aerial robotics. In 2016 International Conference on Unmanned Aircraft Systems (ICUAS)

[22] J. L. Sanchez-Lopez, M. Molina, H. Bavle, C. Sampedro, R. A. Suárez Fernández, and P. Campoy. A multi-layered component-based approach for the development of aerial robotic systems: The aerostack framework. Journal of Intelligent \& Robotic Systems, 2017.

[23] Jose Luis Sanchez-Lopez, Jesús Pestana, Paloma de la Puente, and Pascual Campoy. A reliable open-source system architecture for the fast designing and prototyping of autonomous multi-uav systems: Simulation and experimentation. Journal of Intelligent \& Robotic Systems, 84(1):779-797, 2016.

[24] Fabrizio Schiano, Javier Alonso-Mora, Konrad Rudin, Paul Beardsley, Roland Y. Siegwart, and Bruno Sicilianok. Towards Estimation and Correction of Wind Effects on a Quadrotor UAV. In IMAV 2014.

[25] S. Shen, N. Michael, and V. Kumar. Obtaining liftoff indoors: Autonomous navigation in confined indoor environments. IEEE Robotics Automation Magazine, 20(4):40-48, Dec 2013.

[26] L. N. C. Sikkel, G. C. H. E. de Croon, C. De Wagter, and Q. P. Chu. A novel online model-based wind estimation approach for quadrotor micro air vehicles using low cost mems imus. In 2016 IROS, 2016.

[27] E. J. J. Smeur, G. C. H. E. de Croon, and Q. Chu. Gust disturbance alleviation with incremental nonlinear dynamic inversion. In 2016 IEEE/RSJ International Conference on Intelligent Robots and Systems (IROS), pages 5626-5631, Oct 2016

[28] N. Sydney, B. Smyth, and D. A. Paley. Dynamic control of autonomous quadrotor flight in an estimated wind field. In 52nd IEEE Conference on Decision and Control, pages 3609-3616, Dec 2013.

[29] Donglai Wang, Qiang Lv, Feng Liu, and Keke Wang. Quadrotor longitudinal controller based on 1_1 adaptive control method [j] Journal of Projectiles, Rockets, Missiles and Guidance, 6:012, 2011.

[30] Jiang Wang, Chengyu Cao, Naira Hovakimyan, Richard E. Hindman, and D. Brett Ridgely. L1 adaptive controller for a missile longitudinal autopilot design. 2008.

[31] S. Waslander. Wind disturbance estimation and rejection for quadrotor position control, 20092009. 